COLO-HEP-391

\title{
Instanton Effects in Hadron Spectroscopy in $\mathrm{SU}(2)$ (Lattice) Gauge Theory
}

\author{
Thomas DeGrand, Anna Hasenfratz, and Tamás G. Kovács \\ Department of Physics \\ University of Colorado, Boulder CO 80309-390
}

July 2021

\begin{abstract}
We describe quenched spectroscopy in $S U(2)$ gauge theory using smoothed gauge field configurations. We investigate the properties of quarks moving in instanton background field configurations, where the sizes and locations of the instantons are taken from simulations of the full gauge theory. By themselves, these multi-instanton configurations do not confine quarks, but they induce chiral symmetry breaking.
\end{abstract}

\section{Introduction}

What features of the QCD vacuum are responsible for confinement or for the generation of the observed structure of hadron spectroscopy? This question might, in principle, be answered by lattice simulations of non-Abelian gauge theories. In practice, however, the dominant features of the QCD vacuum as seen in lattice simulations are basically identical to the dominant features of the vacuum of any field theory, be it confining or not: short distance fluctuations of the dynamical variables, whose main effect is simply to renormalize the bare 
parameters of the theory. These fluctuations mask the (presumably) longerdistance features which are the true objects of the question.

This complication has a standard solution in lattice simulations, namely to transform the lattice in a way that reduces short-distance fluctuations, but leaves the long distance properties unchanged. Observables constructed from the new variables correspond to extended, sometimes non-local observables on the original lattice. Examples of these constructions include APE-blocked links [1], used in measurements of the glueball spectrum and string tension, "cooled links" [2] used in many investigations of topology on the lattice, or the use of "smeared operators" [3] in matrix element calculations. The separation of structure in the vacuum into two classes, short distance vs. long distance, is intrinsically ambiguous, and this can contaminate one's results. For example, instantons are localized objects of size about 0.2-0.3 $\mathrm{fm}$, i.e. they are not long distance quantities in the continuum. On the lattice at small lattice spacing they span large distance in lattice units and a local transformation will preserve them. On coarse lattices, however, their size in lattice units is small and a smoothing transformation might lose physically relevant objects. If one wants to study the vacuum by using particular ultraviolet-insensitive observables/transformation, one ought to be certain that they do not alter the long distance behavior one is interested in.

Once a particular lattice transformation reveals the structure of the vacuum, we can ask in what way a particular set of dynamical degrees of freedom is responsible for the dynamics of the system. We would regard the evidence for the importance convincing only, if an approximation to the vacuum which included only the considered degrees of freedom predicted hadronic properties in rough quantitative agreement with those from the full theory.

In this note we concentrate on instanton excitations of the vacuum. Based on phenomenological models, it has been argued that instantons are largely responsible for chiral symmetry breaking and the low energy hadron and glueball spectrum [4, 5]. Instanton liquid models attempt to reproduce the topological content of the QCD vacuum and conclude that hadronic correlators in the instanton liquid show all the important properties of the corresponding full QCD correlators. These models appear to capture the essence of the QCD vacuum, but their derivations involve a number of uncontrolled approximations and phenomenological parameters.

We are motivated to study this problem because of our work on instantons [6], where we devised a method of constructing smoothed gauge configurations whose long distance behavior was identical to the original configurations, but which had tiny short distance fluctuations. We found that most of the action of the smoothed configurations is carried by instantons. But to what extent do 
instantons affect long distance physics such as confinement or hadron structure? We use the smoothed configurations as templates to construct configurations containing only instantons, and then investigate the spectral properties of quarks moving in these background configurations.

Our smoothing mechanism is called "cycling." Imagine beginning with a set of lattice variables $\{V\}$ on a lattice whose spacing is $a$ and lattice size is $L$. The lattice action is a fixed-point action [7] $S^{F P}(U)$. The first part of a cycling step is done by performing an inverse blocking transformation to construct a set of fine lattice variables $\{U\}$ occupying a lattice of lattice spacing $a / 2$ and lattice size $2 L$, by solving the steepest-descent equation

$$
S^{F P}(V)=\min _{\{U\}}\left(S^{F P}(U)+\kappa T(U, V)\right)
$$

Here $\kappa T(U, V)$ is the renormalization group blocking kernel. Now the original lattice occupies one of the 16 sublattices of the fine lattice. Performing an RG blocking transformation from the fine variables to a set of variables based on the sublattice corresponding to the original lattice merely inverts the minimization of Eq. 1. However, if we perform a blocking transformation to a set of coarse variables $\{W\}$ based on one of the other sublattices, the delicate coherence among the fine variables will be broken and the new coarse variables will be strongly ordered on the shortest distance scale (as measured, for example, by the expectation value of the plaquette) while retaining all long distance physics (because they are generated by a RG blocking transformation). This is the second part of the cycling transformation $V_{\mu}(x) \rightarrow U_{\mu}(x) \rightarrow W_{\mu}(x)$. Cycling steps can be iterated, and a few cycling steps can reduce the plaquette to within 0.001 of its free-field value.

One can think of performing spectroscopy on cycled gauge configurations as performing spectroscopy on the original configurations, but using a more complicated fermion action in which the naive gauge connections are replaced by cycled links. This is similar in spirit to the use of "fat links" by the MILC collaboration [8] or the complicated gauge connections of the approximate FP actions developed by one of us [9]. The "cycled fermions" are more insensitive to short distance fluctuations of the gauge fields than the original fermions.

By itself, cycling should not be expected to dramatically improve scaling, since the discretization errors of the underlying free fermion action are unchanged. However, cycling decouples the fermions from short distance fluctuations present in the simulations. (This is in contrast to tadpole improvement [10], as it is usually implemented in simulations, in which couplings are merely rescaled.) For Wilson fermions we expect to see the critical hopping parameter $\kappa_{c}$ closer to $1 / 8$, even at strong coupling. For staggered fermions, we expect to see a dramatic improvement in flavor symmetry restoration, as given by the 
mass difference between the Goldstone and non-Goldstone local pions.

The simulations reported here were performed in $S U(2)$ gauge theory using the FP gauge action of Ref. [6]. We worked at a coupling $\beta=1.5$ (close to the critical coupling for deconfinement at $N_{t}=4$ with string tension $a^{2} \sigma=0.122(10)$ and Sommer radius $\left.r_{0} / a=3.48(2)\right)$. We cycled up to 9 times a set of about 30-40 configurations of size $8^{3} \times 16$ sites. We computed Wilson spectroscopy on one source time slice, and staggered spectroscopy on two time slices, per lattice. The staggered fermions have antiperiodic temporal boundary conditions; the Wilson fermions used periodic boundary conditions. We only performed meson spectroscopy. In this note we concentrate on staggered fermions, since they provide a cleaner signal of the physics of interest.

Fig. 1 shows $a m_{\rho}$ vs. $a m_{\pi}$ for the staggered fermion action, with raw gauge links and 9-cycled links. It appears that cycling does not affect this sector of spectroscopy.

However, the picture is quite different for the non-Goldstone partner of the pion, as shown in Fig. 2 and 3. On the original lattices the two mesons have a mass ratio of about 1.4 . On the 9 -cycled lattices the two pions are degenerate within observational uncertainty.

Note that in Fig. 2 the bare mass needed to produce the same pion mass is much larger on the cycled lattice than on the original lattice. This is quite reasonable given the observed size of fluctuations on the shortest distance scale: put differently, $f_{\pi}^{2} m_{\pi}^{2}=Z m_{q}\langle\bar{\psi} \psi\rangle$ with $Z$ much smaller on the smoothed lattices than on the original ones.

All of these results are anticipated by the "fat link" action of the MILC collaboration [8. There, work is done in $S U(3)$ at $\beta=5.85$, closer to $\beta_{c}\left(N_{t}=\right.$ $5-6)$. The pions in that work begin with $m_{\pi 2} / m_{\pi} \simeq 1.6$, and their smoothing reduces this number to about 1.2. Similar results, using a more complicated blocking procedure, have been reported by J.-F. Lagaë and D. K. Sinclair [11].

The purpose of this exercise is not to test spectroscopy in detail. The important point is that the action of the many-times-cycled configurations is dominated by instantons, and yet the heavy quark potential and hadron spectroscopy are basically unchanged from what we saw on untouched configurations.

We believe that the physical picture we see at this point is very similar to one recently presented by Ivanenko and Negele [12]. These authors calculated the lowest eigenvalues of the Dirac operator and found that the rho and pion contributions to the vector and pseudoscalar correlation functions were dominated by these lowest eigenfunctions. The lowest eigenfunctions are sensitive to 


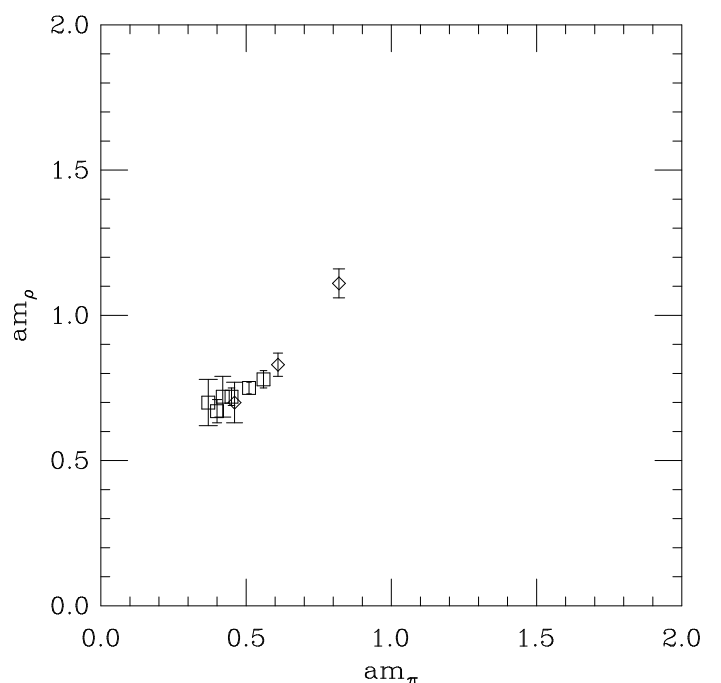

Figure 1: $a m_{\rho}$ vs. $a m_{\pi}$ for the staggered fermion action, with untouched gauge links (diamonds) and 9-cycled links (squares).

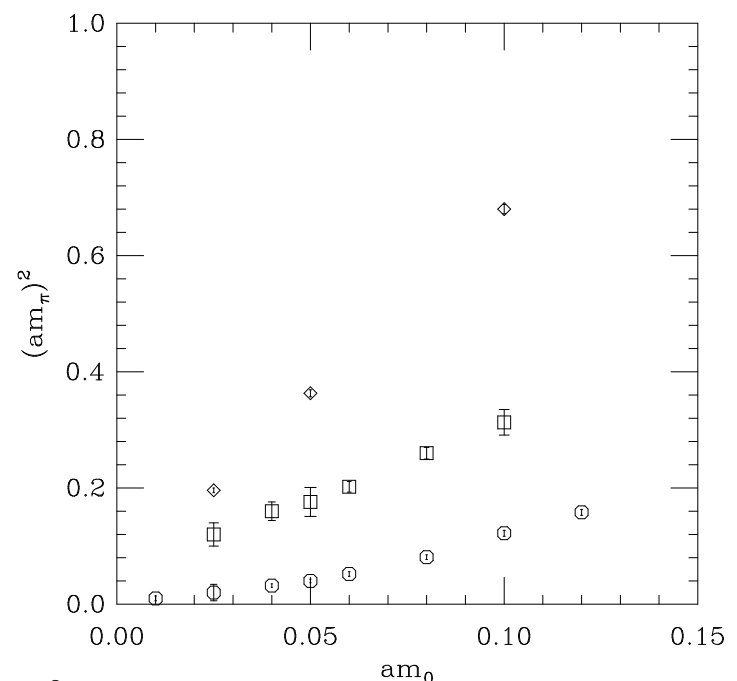

Figure 2: $\left(a m_{\pi}\right)^{2}$ vs. $m_{0}$ the bare quark mass for the staggered action, with raw gauge links (diamonds) and 9-cycled links (squares). The lightest mass in the pseudoscalar channel with instanton background configurations is shown by octagons. 


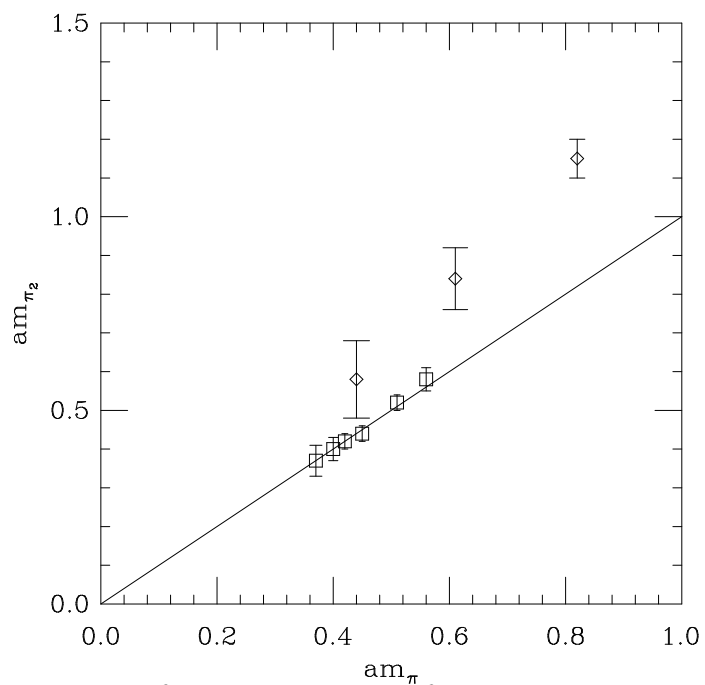

Figure 3: $a m_{\pi_{2}}$ vs. $a m_{\pi}$ for the staggered fermion action, with raw gauge links (diamonds) and 9-cycled links (squares). The straight line is $a m_{\pi_{2}}=a m_{\pi}$.

the positions of the instantons in the background gauge fields.

But these results do not answer the question: Are instantons responsible for long distance physics, or are the structures responsible for long distance physics some other objects carrying low action, which have been preserved by the cycling transformation?

We can test that question by preparing gauge configurations containing only instantons and anti-instantons, and using those configurations as an ensemble for spectroscopy calculations. We do this by identifying the instantons' sizes and locations from many-times cycled configurations, then constructing gauge field configurations appropriate to the superposition of instantons. The instanton identification is always done on the inverse blocked fine lattices, where the positions and sizes of instantons are measured. Then the instanton content is reconstructed on the same size lattice in the following way.

Our starting point is a set of lattice configurations containing single instantons with radii 1.0, 1.5, 2.0...7.0 lattice spacings. These are obtained by discretizing continuum instantons in the singular gauge (see Refs. [13]). In this gauge the vector potential falls off sufficiently rapidly so that in our ensemble the instantons do not overlap significantly and any sensible ansatz can be used to combine their fields. For each instanton found in a given 9-cycled configuration we took the one in our sequence that was closest in size and shifted it to 
the appropriate position. The vector potential of single instantons was approximated by the logarithm of the links. We added the vector potentials of all the instantons found in the given configuration and finally reexponentiated the sum to get the link $\mathrm{SU}(2)$ elements. After the instanton content was reconstructed on the fine lattice, it was blocked and all the measurements were performed on the blocked "coarse" lattices.

Since we have not determined the relative orientation of instantons in group space, in our earlier work we constructed the artificial instanton configurations from "parallel" instantons. In the present work we also built instanton configurations where the instanton orientations are randomly distributed according to the SU(2) Haar measure. This was done by performing a random global (constant in space-time) gauge transformation on each individual lattice instanton before using it to construct the given instanton configuration.

We now compare the three ensembles; the 9 times cycled real configurations, the parallel and the randomly oriented instanton configurations with the instanton sizes and locations exactly reproduced from the 9-cycled configurations. It is interesting to note that the average action per configuration is 11.9 on the 9-cycled, 7.9 on the randomly and 8.4 on the parallelly oriented instanton ensemble (in units of the one-instanton action). The average number of instantons per configuration is 6.7 .

What is the scale of these ensembles? The 9 times cycled configuration has the same lattice spacing as the original lattice by construction. One might want to argue that the physical scale is different for the instanton-background simulations. In instanton liquid models, the physical scale is set by the size and/or separation of the instantons, which in these simulations is derived from the instanton sizes and locations on the original gauge configurations. So we believe that the most natural choice for a physical scale in these configurations is the same as on the original configurations.

In Fig. 1 the heavy-quark potentials obtained from the three ensembles are compared. We can conclude that neither the parallel nor the randomly oriented instantons confine. In fact the potentials produced by the parallel and randomly oriented instanton ensembles are hardly distinguishable. It is true that in the real configurations instantons are probably neither parallel nor completely randomly oriented but since the difference between these two extremes is so small, it is unlikely that any special orientation arrangement can produce substantially different results. It seems that instantons by themselves are not responsible for confinement.

We have also computed spectroscopy on the two types of instanton ensembles using both staggered and Wilson fermions. The dominant feature of both 


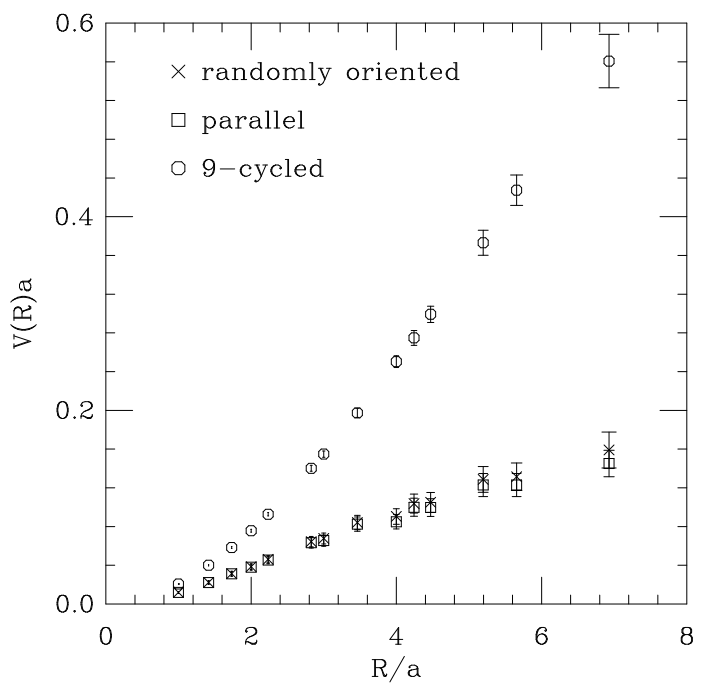

Figure 4: The heavy-quark potential measured on the 9 times cycled real configurations (octogons), the randomly (crosses) and the parallelly (squares) oriented instantons.

spectroscopy calculation is that the quarks are deconfined. This is seen most easily in the staggered fermion spectroscopy.

Consider the pseudoscalar propagator of the 9-cycled configurations, shown for one quark mass in Fig. 5 . It looks like any generic lattice pseudoscalar, a more-or-less pure hyperbolic cosine with no oscillations. The staggered fermion pseudoscalar propagators on instanton background fields are quite different: they show the characteristic sawtooth pattern of free antiperiodic fermions. This is shown in Fig. 6 .

For Wilson fermions, the correlators are qualitatively similar to the correlators of free $q \bar{q}$ pairs on the instanton configurations. A single-exponential fit to the pseudoscalar and vector channels gives masses which fall to zero as the hopping parameter approaches $1 / 8$ and the vector and pseudoscalar remain degenerate.

And yet, there is physics beyond deconfinement in these configurations. Fig. 0 shows $\langle\bar{\psi} \psi\rangle$ for staggered fermions on the 9-cycled and in instanton background configurations. $\langle\bar{\psi} \psi\rangle$ in instanton background tracks the value of $\langle\bar{\psi} \psi\rangle$ measured on the 9-cycled configurations quite closely, down to small quark mass. It appears that the instantons, present in equilibrium gauge field configurations of the QCD vacuum generated by Monte Carlo, are breaking chiral symmetry by themselves. This effect is a cornerstone of instanton-liquid models of hadron 


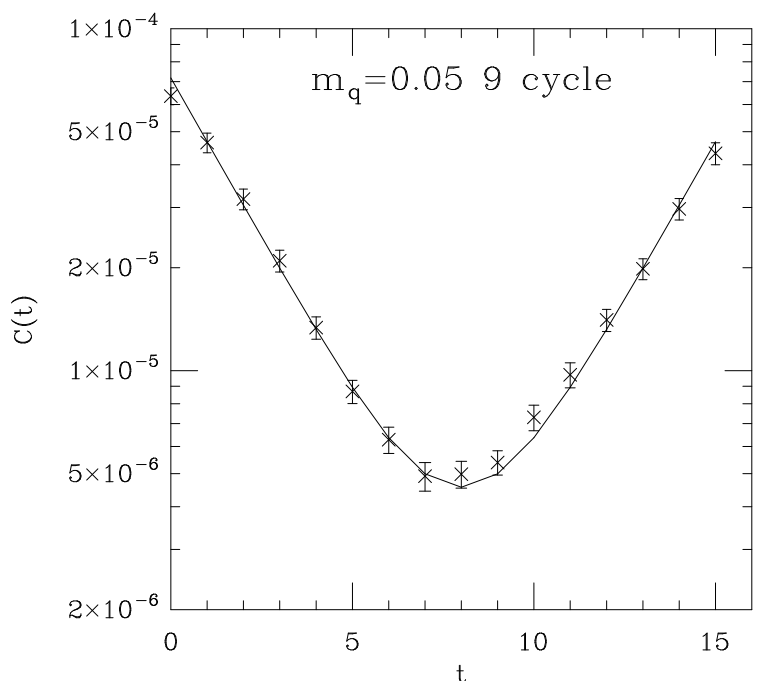

Figure 5: The pseudoscalar propagator from 9-cycled configurations, with staggered fermions of bare mass $a m_{0}=0.05$.

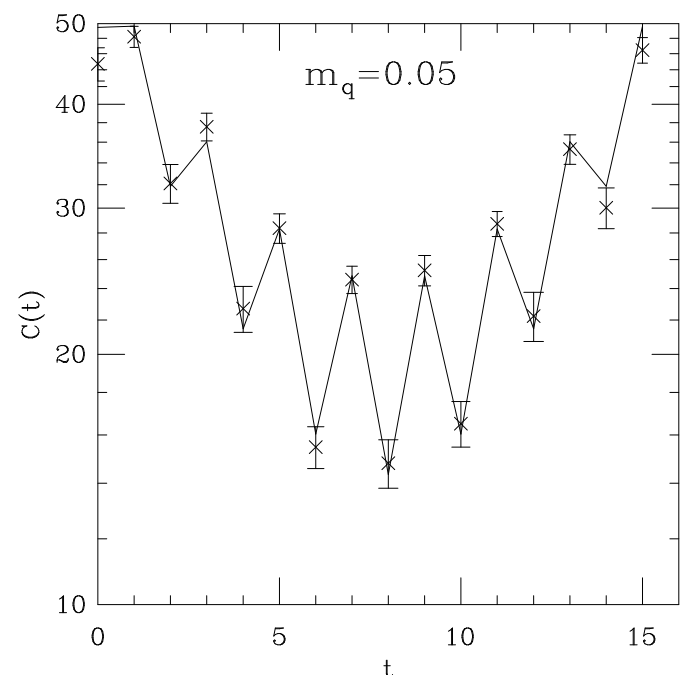

Figure 6: The pseudoscalar propagator in (randomly rotated) instanton background configurations, with staggered fermions of bare mass $a m_{0}=0.05$. The curve is a fit to a single propagating particle plus the $q \bar{q}$ branch cut. 


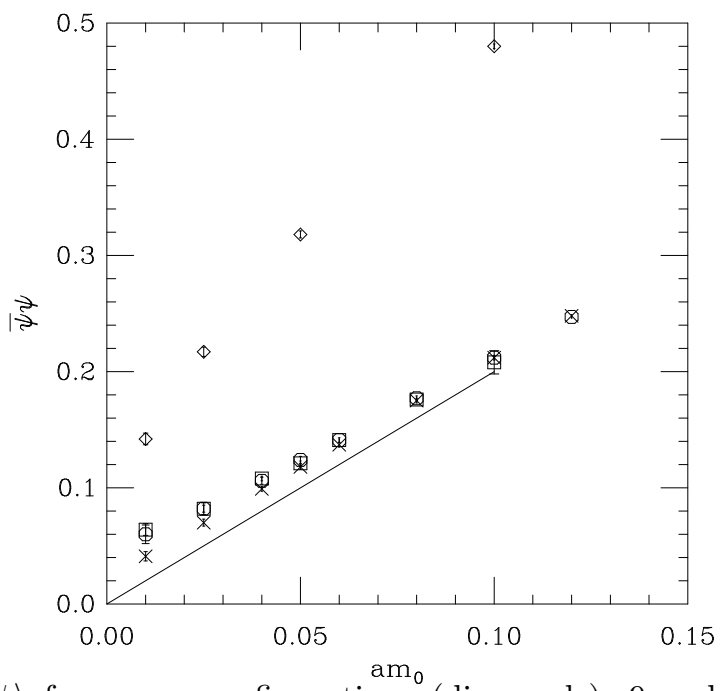

Figure 7: $\langle\bar{\psi} \psi\rangle$ from raw configurations (diamonds), 9-cycled configurations (squares), and instanton-background configurations which are parallel (crosses) and randomly oriented (octagons), vs. bare quark mass $a m_{0}$. The line shows the free-field value, $2 m_{0}$.

structure.

If $\langle\bar{\psi} \psi\rangle$ is nonzero, one expects the spectrum contains a would-be Goldstone boson (the pion) in addition to massive quarks and (possibly) other resonances. To test this hypothesis, we fit the pseudoscalar correlator to two terms: a pure hyperbolic cosine (a pole in the frequency plane), plus a $q \bar{q}$ branch cut, with the quark mass as a parameter. We used the analytic expression for the branch cut (expressed as a momentum and frequency mode sum), with the appropriate boundary conditions and source used in the simulations (computed simply by measuring the correlators on trivial background gauge configurations).

Since we have only 30 instanton configurations, the fits for the bound state masses are quite poor. The signal in the oriented instanton background is quite noisy and the fit masses are not well determined. The resulting (squared) pion mass is shown in Fig. 8. It is quite small and appears to be decreasing as the bare mass vanishes. We cannot tell if it vanishes at zero bare quark mass. The signal in the random instanton background is much larger and more stable. We clearly see a light mass which decreases towards zero as the quark mass vanishes.

The quark mass in the randomly oriented instanton background is also determined by the fit. The free correlator depends weakly (quadratically) on the 


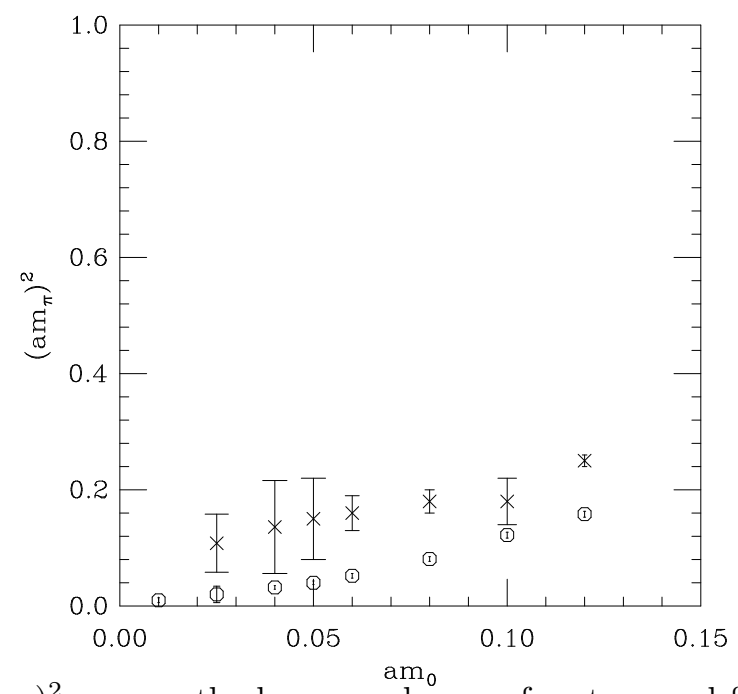

Figure 8: $\left(a m_{\pi}\right)^{2}$ vs. $m_{0}$ the bare quark mass for staggered fermions in background instanton configurations, with parallel (crosses) and randomly rotated instantons (octagons).

precise value of the quark mass when it is very small. At $a m_{0}=0.10$ we fit $a m_{q}=0.109(2), 0.105(3)$ at $a m_{0}=0.08$, and 0.08(4) at $a m_{0}=0.04$. The quark mass is less than half the rho mass-and less than half the pion mass- (as measured on the 9-cycled lattices).

Are there other bound states? Only for the randomly rotated instanton configurations could we see a convincing pseudoscalar, and so we restricted our analysis to that data set. We again attempted to fit to a single resonance plus a $q \bar{q}$ continuum. Of course, this assumption is questionable.

Even in the usual (confined) case, the other meson channels are dominated by two relatively light particles of opposite parity. We are attempting to fit the correlator in the instanton background field to a free quark continuum (which oscillates in time) and only a single resonance. This may be incorrect. The correlator might have contributions from two opposite parity bound states and a $q \bar{q}$ continuum, or no continuum, or no resonances. So we may just be fitting the effects of a large number of excited states, or of interactions between free quarks, to a single exponential, and (incorrectly) interpreting our results as evidence for a resonance. The fits are not of high quality and our results should not be taken too seriously: In the "SC" channel (saturated by the $\pi_{2}$ and scalar mesons in the confined phase) we saw a light bound state whose mass roughly tracked the mass of the (presumed) pion resonance in the pseudoscalar channel, falling from 
about $a m\left(\pi_{2}\right)=0.44$ at $a m_{0}=0.12$ to about $a m\left(\pi_{2}\right)=0.18$ at $a m_{0}=0.04$. In the PV and VT channels, a state with a mass 0.7-0.6 appears, in addition to the free $q \bar{q}$ continuum. This state has about the same mass as the vector meson in the confined system, at an equivalent pseudoscalar mass. However, the dominant feature of all these channels is still the free $q \bar{q}$ continuum, with fitted quark masses of $a m_{q}=0.1$ or lower. The energy of the free $q \bar{q}$ continuum is always lower than the mass of any (presumed) resonance.

Our work shows that instantons have broken chiral symmetry and probably that a light pion has been generated. However, instantons do not confine, and the properties of quarks propagating in instanton background field are quite different from those of the full theory.

All this work is done at large lattice spacing of about $0.14 \mathrm{fm}$. Our work [6] has shown that the typical size of instantons in $\mathrm{SU}(2)$ gauge theory is about $\rho \simeq 0.2 \mathrm{fm}$, and it may well be that staggered fermions do not couple well to such small (in units of the lattice spacing) structures. Thus it would be interesting to repeat this work at smaller lattice spacing. Does the mass scale of the free quark continuum ever rise above the mass scale of any hadronic bound states?

It would also be very interesting to repeat these studies for $S U(3)$ gauge theory. However, the cycling transformation is simply too expensive to use for large volumes in $S U(3)$. It may be that it is possible to replace cycling by some kind of local averaging of the fields on the original lattice, tuned to

preserve medium-scale physics, including topological structures larger than some minimum size. This construction is presently under study.

\section{Acknowledgements}

We would like to thank the Colorado High Energy experimental groups for allowing us to use their work stations. This work was supported by the U.S. Department of Energy.

\section{References}

[1] M. Falcioni, M. Paciello, G. Parisi, B. Taglienti, Nucl. Phys. B251[FS13] (1985) 624. M. Albanese, et al. Phys. Lett. B192 (1987) 163.

[2] D. J. R. Pugh, M. Teper, Phys. Lett. B218 (1989) 326. 
[3] Cf. G. Kilcup, R. Gupta, and S. Sharpe, hep-lat/9707006.

[4] D. Diakanov, Lectures at the Enrico Fermi School in Physics, Varenna, 1995, hep-ph/9602375.

[5] T. Schäfer and E. V. Shuryak, "Instantons in QCD," hep-ph/9610451.

[6] T. DeGrand, A. Hasenfratz, T. Kovacs, hep-lat/9705009.

[7] P. Hasenfratz and F. Niedermayer, Nucl. Phys. B414 (1994) 785; T. DeGrand, A. Hasenfratz, P. Hasenfratz, F. Niedermayer, Nucl. Phys. B454 (1995) 587, Nucl. Phys. B454 (1995) 615.

[8] MILC collaboration, hep-lat/9609036.

[9] T. DeGrand, poster presented at Lattice 97, hep-lat/9709052.

[10] Indeed, the original application of the "tadpole improvement" program to calculations of lattice quantities was to account for the dominant effects of quantum fluctuations on short-distance quantities via an appropriatelydefined perturbative expansion. See G. Parisi, in the Proceedings of the XX International Conference on High Energy Physics, L. Durand and L. Pondrom, eds., American Institute of Physics, p. 1531 and G. P. Lepage and P. Mackenzie, Phys. Rev. D48, 2250 (1993).

[11] J.-F. Lagaë and D. K. Sinclair, talk presented at Lattice '97, heplat/9709035.

[12] J. W. Negele, hep-lat/9709129; T. L. Ivanenko and J. W. Negele, talk presented at Lattice '97, hep-lat/9709130.

[13] D.J.R. Pugh, and M. Teper, Phys. Lett. B224 (1989) 159; T. DeGrand, A. Hasenfratz, and D. Zhu, Nucl. Phys. B475 (1996) 321. 